\title{
Preoperative prognostic nutritional index predicts short- and long-term outcomes after liver resection in patients with hepatocellular carcinoma
}

\author{
YU SAITO, SATORU IMURA, YUJI MORINE, TETSUYA IKEMOTO, \\ SHINICHIRO YAMADA and MITSUO SHIMADA \\ Department of Surgery, Tokushima University, Tokushima 770-8503, Japan
}

Received May 16, 2020; Accepted October 14, 2020

DOI: $10.3892 / \mathrm{ol} .2020 .12414$

\begin{abstract}
The prognostic nutritional index (PNI) is one of the immune parameters calculated on the basis of the serum albumin and the total lymphocyte count. The aim of the present study was to investigate the prognostic significance of the PNI for short- and long-term outcomes after liver resection for patients with hepatocellular carcinoma (HCC). Data from 162 surgically treated patients with HCC (without any previous treatment) were retrospectively analyzed. The cutoff value of preoperative PNI was 45.0, which was calculated by a receiver operating characteristic curve for predicting the recurrence of $\mathrm{HCC}$ after liver resection. Patients were divided into low $(n=86)$ and high $(n=76)$ PNI groups. In short-term outcomes, patients in the low PNI group were more likely to experience postoperative complications compared with those in the high PNI group. The 5-year disease-free survival (DFS) rate in the low PNI group was significantly lower compared with that in the high PNI group (20.5\% vs. $48.7 \%)$. In the multivariate analysis, a low PNI was an independent prognostic factor for DFS (HR, 1.65; 95\% CI, 1.00-2.71). In conclusion, the preoperative PNI may be a prognostic factor for evaluating short- and long-term outcomes after liver resection in patients with HCC.
\end{abstract}

Correspondence to: $\mathrm{Dr} \mathrm{Yu}$ Saito, Department of Surgery, Tokushima University, 3-18-15 Kuramoto-cho, Tokushima 770-8503, Japan

E-mail: saito.yu.1001@tokushima-u.ac.jp

Abbreviations: $\mathrm{PNI}$, prognostic nutritional index; Hx, liver resection; HCC, hepatocellular carcinoma; NLR, neutrophil-to-lymphocyte ratio; ALRI, aspartate aminotransferase-to-lymphocyte ratio; AFP, $\alpha$-fetoprotein; DCP, des-gamma-carboxy prothrombin

Key words: prognostic nutritional index, surgical outcomes, hepatocellular carcinoma

\section{Introduction}

Hepatocellular carcinoma (HCC) is one of the most common solid tumors worldwide, and the third most common cause of cancer-related death (1). For liver resection ( $\mathrm{Hx}$ ) in patients with HCC, the perioperative complications and mortality have improved in recent years from $30 \%$ to $<5 \%$ in high-volume centers $(2,3)$; however, it is crucial to manage patients with various comorbidities. $\mathrm{Hx}$ is still associated with a $40 \%$ morbidity rate, with post-hepatectomy liver failure (PHLF) being the most lethal complication (4). Furthermore, the recurrence rate after curative $\mathrm{Hx}$ for HCC is still higher compared with that for other digestive organ cancers, suggesting that it is also important to assess the risk factors for recurrence following curative $\mathrm{Hx}$.

The prognostic nutritional index (PNI) was first established in 1980 in order to predict perioperative risk for gastrointestinal surgeries (5). However, the calculation method was highly complicated as it used a number of parameters such as serum albumin (Alb), triceps skinfold, transferrin and delayed hypersensitivity skin testing. A simpler modified PNI using serum Alb and total lymphocyte count (TLC) alone was reported 4 years later by Onodera et al (6), and the modified PNI has been widely used for perioperative risk assessment. In addition to perioperative risk prediction, the PNI has also been reported to be associated with long-term prognosis of various types of cancer after curative treatment, such as lung (7), ovarian (8), cervical (9), gastric (10) and colorectal (11) cancer.

In $\mathrm{Hx}$ for $\mathrm{HCC}$, the preoperative PNI is associated with liver function markers such as the albumin-bilirubin grade and predicts short-term outcomes within the Milan criteria (12). A previous study has been reported that the preoperative PNI predicts long-term prognosis after $\mathrm{Hx}$ in only early Barcelona clinic liver cancer stage HCC (13). To the best of our knowledge, there have been no reports on the use of the preoperative PNI for predicting both short- and long-term outcomes in early and advanced stages of HCC.

The present study aimed to compare three types of immune parameters, namely the PNI, neutrophil-to-lymphocyte ratio (NLR) and aspartate aminotransferase (AST)-to-lymphocyte ratio (ALRI), as predictors of short- and long-term outcomes following Hx. Furthermore, the aim of the present study was to 
determine the prognostic significance of the preoperative PNI for short- and long-term outcomes after $\mathrm{Hx}$ for patients with all stages of HCC.

\section{Materials and methods}

Patients. Among 229 patients who underwent Hx at Tokushima University Hospital (Tokushima, Japan) between January 2006 and December 2014, 162 patients were enrolled in the present study. The inclusion criteria were as follows: i) Primary Hx; ii) no other treatments before $\mathrm{Hx}$; and iii) availability of the follow-up data. The study was approved by Tokushima University Hospital Ethics Committee, and the experiments were carried out in accordance with the approved guidelines (Tokushima Clinical Trial Management System Number, 3215). All patients involved in the study signed the informed consent form and agreed to participate.

Preoperative immune parameters. Blood samples were collected prior to Hx. The PNI was calculated as the sum of Alb level and $0.005 x$ lymphocyte count. The NLR was calculated by dividing neutrophil count by lymphocyte count. The ALRI was calculated by dividing AST by lymphocyte count. The cutoff value of the PNI, NLR and ALRI was 45, 2.3 and 30.8, respectively, calculated by a receiver operating characteristic curve for predicting recurrence after Hx (Fig. S1).

Assessment offat mass (FM) and skeletal muscle mass (SMM). Preoperative FM and SMM was determined from CT using Synapse Vincent ${ }^{\circledR}$ (Version 4, Fujifilm Corporation). Visceral FM $\left(\mathrm{cm}^{2}\right)$, subcutaneous FM $\left(\mathrm{cm}^{2}\right)$ and SMM/height $\left(\mathrm{cm}^{2} / \mathrm{m}^{2}\right)$ were automatically calculated.

Short-term outcomes after Hx. The short-term outcomes were defined as follows: i) Operative factors (surgical procedures, surgery time, blood loss and the presence or absence of transfusion); and ii) postoperative factors (the presence or absence of complications and hospital stay duration) according to our previous studies $(14,15)$. The '50-50 criteria' introduced by Balzan et al (16) was used as to determine postoperative liver failure. Postoperative complications of grade III or IV in the Clavien-Dindo classification $(14,15)$ were recorded in the present study.

Follow-up after $H x$. The mean follow-up period was 2.48 years (0.02-7.96 years). Monthly follow-up was conducted by ultrasonography and assessment of the tumor markers $\alpha$-fetoprotein (AFP), des-gamma-carboxy prothrombin (DCP) and AFP-L3. Dynamic CT scan and gadoliniumethoxybenzyl-diethylene-triaminepentaacetic acid-enhanced MRI were conducted at 3 and 6 months post-surgery. Recurrence was defined as the appearance of new lesions with radiological features typical of HCC confirmed by at least two imaging methods. Overall survival (OS) was defined as the time between $\mathrm{Hx}$ and death from any cause, and disease-free survival (DFS) was defined as the time between $\mathrm{Hx}$ and recurrence.

Statistical analysis. Continuous variables are presented as the mean \pm standard deviation. Statistical analysis was performed using SPSS Version 21.0 statistical software (IBM Corp.).
Associations between the PNI and patient clinicopathological characteristics were analyzed using the $\chi^{2}$ and Mann-Whitney $\mathrm{U}$ tests. Survival curves were drawn using the Kaplan-Meier method and compared with the log-rank test. Univariate analysis of differences between two groups was also determined by log-rank tests. Multivariate analysis was performed based on the Cox proportional hazards regression model. The factors included for analyses were patient age ( $>70$ years vs. 70 years), sex (male vs. female), hepatitis B antigen (absent vs. present), hepatitis $\mathrm{C}$ virus antibody (absent vs. present), AFP (>200 ng/ml vs. $\geq 200 \mathrm{ng} / \mathrm{ml}$ ), DCP (<400 mAU/ml vs. $\geq 400 \mathrm{mAU} / \mathrm{ml}$ ), tumor number (single vs. multiple), tumor size ( $<3 \mathrm{~cm}$ vs. $\geq 3 \mathrm{~cm}$ ), tumor differentiation (well- and moderately vs. poorly differentiated), portal invasion (absent vs. present), stage (I and II vs. III and IV) and PNI (high vs. low). $\mathrm{P}<0.05$ was considered to indicate a statistically significant difference.

\section{Results}

Comparison of immune parameters among the PNI, NLR and ALRI. Table I presents the comparison in of short-term (postoperative factors; frequency of postoperative complications and hospital stays) and long-term (OS and DFS) outcomes among patients divided into groups based on the PNI, NLR or ALRI. Postoperative factors alone are presented as short-term outcomes for simplicity. The PNI alone was associated with short-term outcome, and both the PNI and NLR were reliable parameters for predicting the long-term outcomes (Table I).

Associations between the preoperative PNI and clinicopathological characteristics. Associations between the preoperative PNI values and clinicopathological characteristics are presented in Table II. A low PNI was significantly associated with an older age, female sex and impaired liver function, such as high AST and indocyanine green retention test values. Among the tumor factors, a low PNI only exhibited a tendency towards the presence of microscopic portal vein invasion. In addition, a low PNI was significantly associated with a low SMM (sarcopenia).

Preoperative PNI and short-term outcomes. No significant differences were observed in the operative procedures between the low and high PNI groups. Blood loss was significantly higher in the low PNI group compared with that in the high PNI group. The low PNI group had significantly longer hospital stays after Hx compared with those in the high PNI group (Table III).

Preoperative PNI and long-term outcomes. Patients in the low PNI group exhibited a tendency towards a lower OS rate (Fig. 1A). In addition, patients in the low PNI group had a significantly poorer DFS compared with that in the high PNI group (Fig. 1B). In the univariate analysis of DFS, high AFP, high DCP, multiple tumors, the presence of microvascular invasion, advanced stage and low PNI were identified as indicators of a poor prognosis. The results of the multivariate analysis revealed that high AFP, multiple tumors and a low PNI were independent prognostic factors for DFS (Table IV). Regarding the recurrence patterns, no significant differences were observed between the low and high PNI groups (Fig. 2). 
Table I. Comparison of immune parameters in short- and long-term outcomes.

\begin{tabular}{|c|c|c|c|c|c|c|c|c|}
\hline \multirow[b]{2}{*}{ Parameter } & \multicolumn{2}{|c|}{$\begin{array}{l}\text { Incidence of } \\
\text { complications }\end{array}$} & \multicolumn{2}{|c|}{ Hospital stay } & \multicolumn{2}{|c|}{ 3-year OS } & \multicolumn{2}{|c|}{ 3-year DFS } \\
\hline & $\%$ & P-value & Days & P-value & $\%$ & P-value & $\%$ & P-value \\
\hline PNI & & 0.12 & & $<0.01^{\mathrm{a}}$ & & 0.06 & & $0.03^{\mathrm{a}}$ \\
\hline$<45.0$ & 8.2 & & $31 \pm 34$ & & 68.4 & & 38.2 & \\
\hline$\geq 45.0$ & 2.7 & & $17 \pm 9$ & & 79.1 & & 53.7 & \\
\hline NLR & & 0.98 & & 0.13 & & $0.01^{\mathrm{a}}$ & & $0.04^{\mathrm{a}}$ \\
\hline$<2.3$ & 5.7 & & $21 \pm 16$ & & 83.9 & & 49.5 & \\
\hline$\geq 2.3$ & 5.6 & & $27 \pm 33$ & & 65.7 & & 40.5 & \\
\hline ALRI & & 0.87 & & 0.67 & & 0.25 & & 0.25 \\
\hline$<30.8$ & 6.5 & & $23 \pm 34$ & & 77.8 & & 51.6 & \\
\hline$\geq 30.8$ & 5.8 & & $25 \pm 18$ & & 71.5 & & 41.5 & \\
\hline
\end{tabular}

${ }^{a} \mathrm{P}<0.05$. PNI, prognostic nutritional index; NLR, neutrophil-to-lymphocyte ratio; ALRI, aspartate aminotransferase-to-lymphocyte ratio.
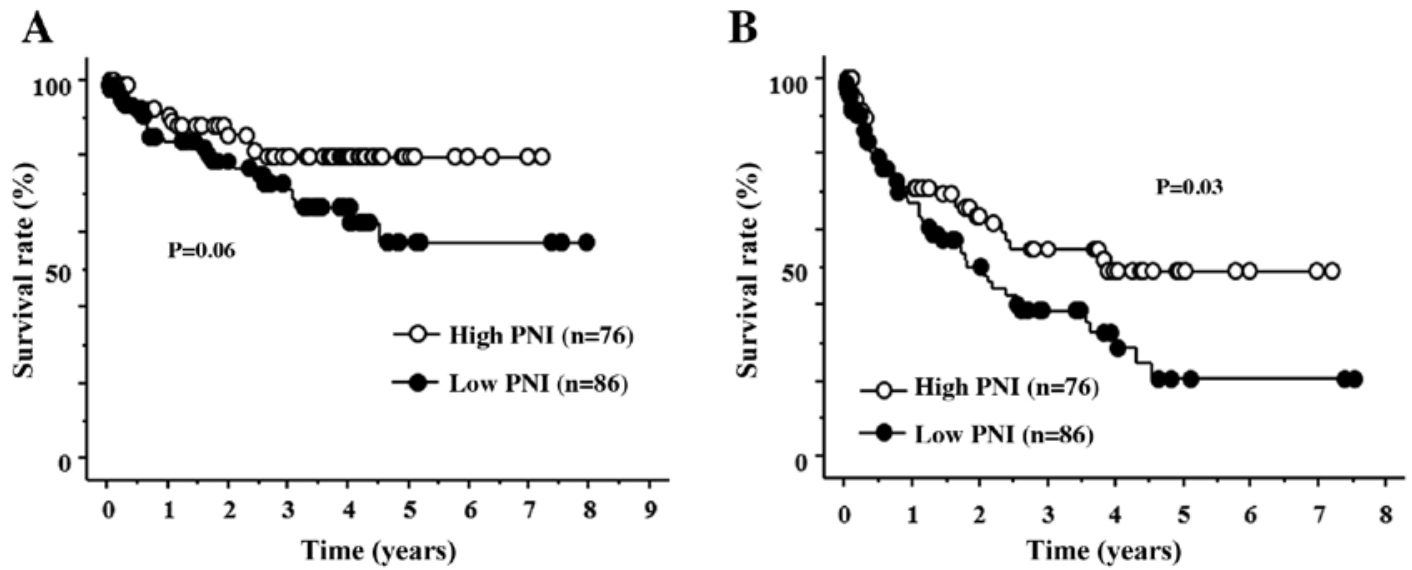

Figure 1. Long-term survival of patients with hepatocellular carcinoma. (A) Overall survival. Patients in the low PNI group tended to exhibit a poorer prognosis compared with those in the high PNI group. (B) Disease-free survival. Patients in the low PNI group exhibited a significantly poorer prognosis compared with those in the high PNI group (5-year survival, 20.5 vs. 48.7\%). PNI, prognostic nutritional index.
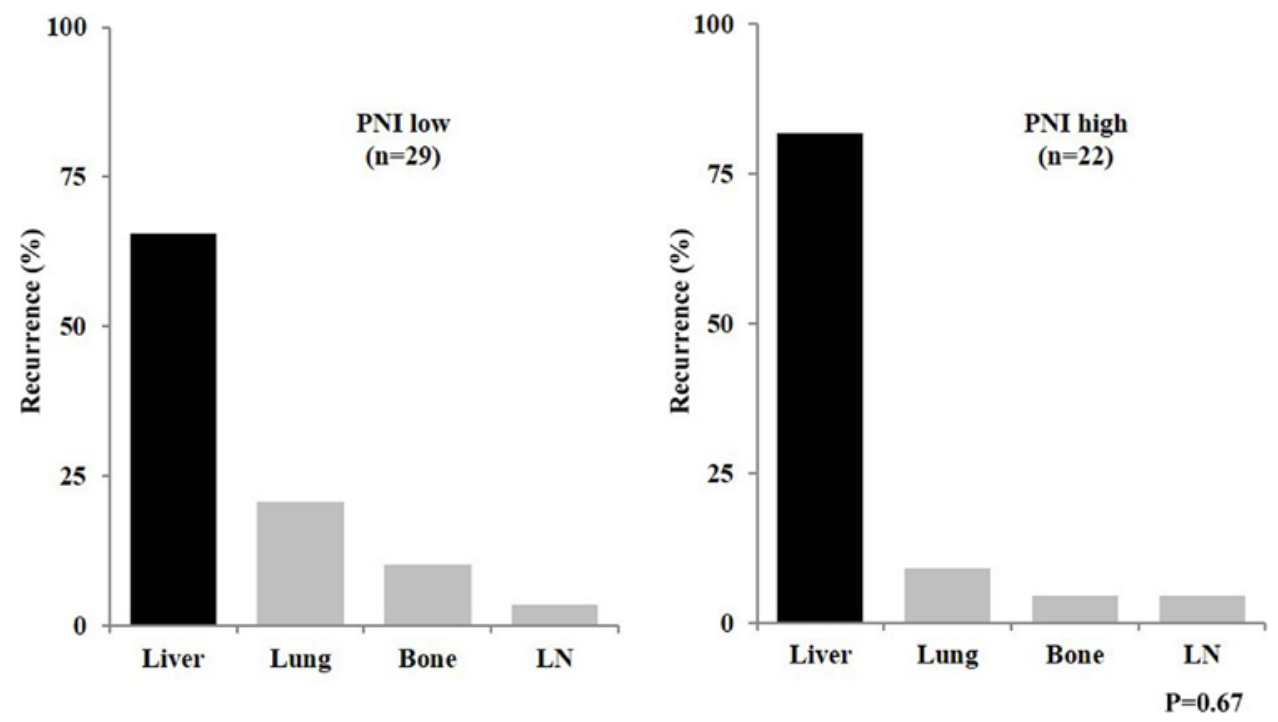

Figure 2. Recurrence patterns. No significant differences were observed in recurrence patterns between patients in the low and high PNI groups. PNI, prognostic nutritional index; LN, lymph node. 
Table II. Associations between the preoperative PNI and patient clinicopathological characteristics.

\begin{tabular}{|c|c|c|c|}
\hline \multirow[b]{2}{*}{ Factor } & \multicolumn{2}{|c|}{ Preoperative PNI } & \multirow[b]{2}{*}{ P-value } \\
\hline & $<45(\mathrm{n}=86)$ & $\geq 45(\mathrm{n}=76)$ & \\
\hline Age, years, mean \pm SD & $70 \pm 10$ & $60 \pm 10$ & $<0.01^{\mathrm{a}}$ \\
\hline Sex & & & 0.03 \\
\hline Male & 57 & 62 & \\
\hline Female & 29 & 14 & \\
\hline Hepatitis B antigen & & & 0.66 \\
\hline Absent & 67 & 57 & \\
\hline Present & 19 & 19 & \\
\hline Hepatitis $\mathrm{C}$ virus antibody & & & 0.34 \\
\hline Absent & 48 & 48 & \\
\hline Present & 38 & 28 & \\
\hline Aspartate aminotransferase, IU/I, mean \pm SD & $60 \pm 36$ & $42 \pm 24$ & $<0.01^{\mathrm{a}}$ \\
\hline Prothrombin, $\%$, mean \pm SD & $100 \pm 17$ & $103 \pm 22$ & 0.31 \\
\hline Total bilirubin, $\mathrm{mg} / \mathrm{dl}$, mean $\pm \mathrm{SD}$ & $0.9 \pm 0.4$ & $0.8 \pm 0.3$ & 0.19 \\
\hline Indocyanine green retention test, $\%$, mean \pm SD & $16 \pm 10$ & $11 \pm 8$ & $<0.01^{\mathrm{a}}$ \\
\hline Platelet count, $\times 10^{4}$, mean $\pm \mathrm{SD}$ & $21 \pm 32$ & $20 \pm 14$ & 0.79 \\
\hline Tumor size, $\mathrm{cm}$, mean $\pm \mathrm{SD}$ & $4.9 \pm 4.5$ & $4.1 \pm 2.7$ & 0.18 \\
\hline Tumor number & & & 0.46 \\
\hline Single & 60 & 57 & \\
\hline Multiple & 26 & 19 & \\
\hline Portal vein invasion & & & 0.06 \\
\hline Absent & 62 & 65 & \\
\hline Present & 24 & 11 & \\
\hline Intrahepatic metastasis & & & 0.65 \\
\hline Absent & 79 & 70 & \\
\hline Present & 7 & 6 & \\
\hline Tumor differentiation & & & 0.47 \\
\hline Well- or moderately differentiated & 17 & 19 & \\
\hline Poorly differentiated & 69 & 57 & \\
\hline Stage & & & 0.73 \\
\hline I and II & 57 & 53 & \\
\hline III and IV & 29 & 23 & \\
\hline $\mathrm{AFP}, \mathrm{ng} / \mathrm{ml}$, mean $\pm \mathrm{SD}$ & $4,385 \pm 22,223$ & $1,350 \pm 5,256$ & 0.25 \\
\hline $\mathrm{DCP}, \mathrm{mAU} / \mathrm{ml}$, mean $\pm \mathrm{SD}$ & $9,359 \pm 32,458$ & $3,172 \pm 13,090$ & 0.22 \\
\hline $\mathrm{BMI}$, mean $\pm \mathrm{SD}$ & $22 \pm 3$ & $23 \pm 3$ & 0.43 \\
\hline Visceral FM, $\mathrm{cm}^{2}$, mean $\pm \mathrm{SD}$ & $76 \pm 47$ & $87 \pm 47$ & 0.11 \\
\hline Subcutaneous FM, $\mathrm{cm}^{2}$, mean $\pm \mathrm{SD}$ & $108 \pm 77$ & $108 \pm 64$ & 0.97 \\
\hline $\mathrm{SMM} /$ height, $\mathrm{cm}^{2} / \mathrm{m}^{2}$, mean $\pm \mathrm{SD}$ & $51 \pm 13$ & $56 \pm 15$ & $0.03^{\mathrm{a}}$ \\
\hline
\end{tabular}

${ }^{\mathrm{a}} \mathrm{P}<0.05$. PNI, prognostic nutritional index; FM, fat mass; SMM, skeletal muscle mass; AFP, $\alpha$-fetoprotein; DCP, des-gamma-carboxy prothrombin.

\section{Discussion}

In the present study, a number of immune parameters including the PNI, NLR and ALRI were compared in terms of short- and long-term outcomes after $\mathrm{Hx}$ in patients with HCC. In addition to the prediction of outcomes following $\mathrm{Hx}$, the PNI was significantly associated with a low SMM. Previous studies have reported similar benefits of the PNI, especially in the prediction of long-term outcomes after Hx. For example, the preoperative $(13,17)$ and postoperative (18) PNIs have been reported to be prognostic factors for OS, especially in early-stage HCC. Furthermore, a meta-analysis has also demonstrated that preoperative PNI is a prognostic marker for long-term survival after not only $\mathrm{Hx}$, but also TACE or 
Table III. Associations between the preoperative PNI and short-term outcomes.

A, Operative factors

\begin{tabular}{lcc}
\hline & \multicolumn{2}{c}{ Preoperative PNI } \\
\cline { 2 - 3 } Factor & $<45(\mathrm{n}=86)$ & $\geq 45(\mathrm{n}=76)$ \\
\hline Procedure & & 17 \\
Hr0 & 29 & 25 \\
S & 4 & 18 \\
1 & 22 & 15 \\
2 & 10 & 1 \\
3 & 1 & $318 \pm 85$ \\
Surgery time, min, mean \pm SD & $328 \pm 93$ & $253 \pm 224$ \\
Blood loss, m, mean \pm SD & $394 \pm 433$ & 0.65 \\
Transfusion & & 63 \\
Absent & 75 & 12 \\
Present & 11 & 0.48 \\
\hline
\end{tabular}

B, Postoperative factors

Preoperative PNI

Factor

$<45(\mathrm{n}=86)$

$\geq 45(n=76)$

P-value

Complications of CD grade III or IV

Absent

79

0.12

Present

79

74

Liver failure

7

Intra-abdominal abscess

3

3

Resistant ascites

1

Hospital stay, days, mean \pm SD

$31 \pm 34$

2

0

0.10

1

0.37

1

0.92

$17 \pm 9$

$<0.01^{\mathrm{a}}$

${ }^{a} \mathrm{P}<0.05$. PNI, prognostic nutritional index; $\mathrm{CD}$, Clavien-Dindo classification; $\mathrm{Hr} 0$, partial resection; Hr S, subsegmentectomy; Hr 1, segmentectomy; Hr 2, lobectomy; Hr 3, trisectionectomy.

Table IV. Univariate and multivariate analysis of disease-free survival.

\begin{tabular}{lrcrr}
\hline & \multicolumn{2}{c}{ Univariate } & \multicolumn{2}{c}{ Multivariate } \\
\cline { 2 - 5 } Factor & P-value & Hazard ratio & $95 \%$ CI \\
\hline $\mathrm{AFP} \geq 200 \mathrm{ng} / \mathrm{ml}$ & $<0.01^{\mathrm{a}}$ & 1.77 & $1.00-3.13$ & $0.04^{\mathrm{a}}$ \\
$\mathrm{nCP} \geq 400 \mathrm{mAU} / \mathrm{ml}$ & $0.04^{\mathrm{a}}$ & 1.04 & $0.59-1.84$ & 0.89 \\
Multiple tumors & $<0.01^{\mathrm{a}}$ & 2.09 & $1.25-3.51$ & $0.79-2.44$ \\
Portal vein invasion present & $<0.01^{\mathrm{a}}$ & 1.39 & $1.00-2.71$ & 0.25 \\
PNI $<45$ & $0.03^{\mathrm{a}}$ & 1.65 & $0.04^{\mathrm{a}}$
\end{tabular}

${ }^{\mathrm{a}} \mathrm{P}<0.05$. PNI, prognostic nutritional index; AFP, $\alpha$-fetoprotein; DCP, des-gamma-carboxy prothrombin.

non-surgical treatment (19). By contrast, a limited number of studies on the prediction of short-term outcomes after $\mathrm{Hx}$ are currently available. One previous report focused on the prognostic ability of the PNI for postoperative complications after Hx within the Milan criteria (12). Therefore, the novelty of the present study compared with the previous ones was that the prognostic ability of the PNI was analyzed in both shortand long-term outcomes after $\mathrm{Hx}$, that the analysis included 
all stages of HCC, and that the PNI was compared with other immune parameters including the NLR and ALRI.

Previous studies have reported the use of the NLR as a prognostic factor in HCC after curative treatment. The usefulness of the NLR and platelet-to-lymphocyte ratio after transarterial chemoembolization has been demonstrated (20). Another study has reported that the NLR predicted disease progression following intra-arterial therapy of $\mathrm{HCC}$ (21). In the present study, the NLR was a predictor of long-term outcomes after Hx. The molecular mechanism of the increase in the NLR involves a number of factors and remains poorly understood. However, an association between the accumulation of tumor-associated macrophages in HCC and high NLR values has been observed in patients with HCC who underwent $\mathrm{Hx}$ and living donor liver transplantation (22). A high NLR is also associated with high levels of infiltration of tumor-associated macrophages and inflammatory cytokine production in the tumor, such as interleukin-6, interleukin-8 and interleukin-17, which promote systemic neutrophilia $(23,24)$.

In the present study, a low PNI was significantly associated with a longer hospital stays and a poor DFS. In addition, patients with a low PNI exhibited a poor OS. By contrast, the NLR was a good prognostic factor predicting long-term outcomes including both OS and DFS. There were no significant associations between the NLR and short-term outcomes. This suggests that the preoperative PNI may be a prognostic factor for evaluating both short- and long-term outcomes. However, there were insufficient data in the present study to conclude that the preoperative PNI was the most significant prognostic factor for evaluating both short- and long-term outcomes among the PNI, NLR and ALRI.

The PNI value, which is a combination of Alb and the total lymphocyte count, may be used to evaluate the immunological and nutritional aspects of patients undergoing surgery. A previous study has reported that the PNI was established as an indirect measure of a patient's nutritional status, suggesting that the PNI may be associated with postoperative complications (12). The level of Alb and the lymphocyte count have been reported to be closely associated with the induction of the inflammatory response (25). Therefore, the PNI may not only reflect the nutritional status, but also systemic inflammation. In the patient cohort included in the present study, Alb level was also a good prognostic factor for both OS and DFS (data not shown). However, no statistically significant differences were observed between Alb levels and the short-term outcomes (data not shown). The PNI appeared to be a better prognostic factor compared with Alb level alone.

Recently, the lymphocyte-to-C-reactive protein ratio (LCR) was reported as a useful marker for predicting surgical and oncological outcomes (26). The present study also investigated the association between the preoperative LCR and prognosis in the study cohort; a low LCR exhibited a tendency towards an association with OS $(\mathrm{P}=0.08)$, but not with DFS. There was no significant association between the LCR and short-term outcomes (data not shown).

A low PNI was not associated with tumor characteristics such as differentiation, stage or tumor markers in the present study. A low PNI was associated with female sex, older age, a poor liver function and low skeletal muscle levels. The same tendency was observed in previous studies $(13,17)$. We therefore speculate that the PNI may reflect the immune-nutritional condition rather than tumor malignancy. Tumor-infiltrating lymphocytes (TILs) are a specific histological feature of human cancers, reflecting an individual's immunological tumor response (27). Previous studies have reported that TILs may be associated with peripheral blood cells, such as the TLC and absolute neutrophil count $(28,29)$. In esophageal cancer, the PNI and TIL score have been reported to be associated with clinical outcomes (30). Considering the relationship between the PNI and TILs, nutritional status and systemic immune competence may affect patient prognosis through local immune response.

For patients with a low PNI, it is essential to improve their outcomes through perioperative nutritional interventions, e.g. the administration of branched-chain amino acid-enriched nutrient support $(31,32)$. In the present study, the PNI was significantly associated with sarcopenia; thus, further nutritional intervention may be necessary for patients with a low PNI.

In conclusion, the results of the present study demonstrated that a low PNI and sarcopenia reflected a poor nutritional status in patients with HCC. Furthermore, the preoperative PNI was a reliable prognostic factor for evaluating both short- and long-term outcomes after Hx for patients with HCC. Therefore, nutritional intervention may be beneficial for patients with HCC with a low preoperative PNI.

\section{Acknowledgements}

The results of the present study were reported at the Clinical Congress of the American College of Surgeons, October 22-26, 2017, in San Diego, CA, USA.

\section{Funding}

This study was partly supported by the Research Program on Hepatitis from the Japan Agency for Medical Research and Development (grant nos. JP19fk0210048 and JP20fk0210048).

\section{Availability of data and materials}

The datasets used and/or analyzed during the current study are available from the corresponding author on reasonable request.

\section{Authors' contributions}

YS participated in the study design, performed the research and data analysis, and wrote the manuscript. SI participated in the study design and data analysis. YM, TI and MS participated in the study design. SY and MS participated in data analysis. MS also critically revised the manuscript for important intellectual content. All authors read and approved the final manuscript.

\section{Ethics approval and consent to participate}

The study was approved by Tokushima University Hospital Ethics Committee (Tokushima, Japan), and all experiments 
were carried out in accordance with the approved guidelines (Tokushima Clinical Trial Management System Number; 3215). All patients involved in the study signed the informed consent form and agreed to participate.

\section{Patient consent for publication}

Not applicable.

\section{Competing interests}

The authors declare that they have no competing interests.

\section{References}

1. Villanueva A: Hepatocellular Carcinoma. N Engl J Med 380 1450-1462, 2019.

2. Harimoto N, Shirabe K, Yamashita YI, Ikegami T, Yoshizumi T, Soejima Y, Ikeda T, Maehara Y, Nishie A and Yamanaka T: Sarcopenia as a predictor of prognosis in patients following hepatectomy for hepatocellular carcinoma. Br J Surg 100: 1523-1530, 2013.

3. Voron T, Tselikas L, Pietrasz D, Pigneur F, Laurent A, Compagnon P, Salloum C, Luciani A and Azoulay D: Sarcopenia impacts on short- and long-term results of hepatectomy for hepatocellular carcinoma. Ann Surg 261: 1173-1183, 2015.

4. Okuda Y, Taura K, Yoshino K, Ikeno Y, Nishio T, Yamamoto G, Tanabe K, Koyama Y, Hatano E, Tanaka S and Uemoto S: Usefulness of Mac-2 binding protein glycosylation isomer for prediction of posthepatectomy liver failure in patients with hepatocellular carcinoma. Ann Surg 265: 1201-1208, 2017.

5. Buzby GP, Mullen JL, Matthews DC, Hobbs CL and Rosato EF: Prognostic nutritional index in gastrointestinal surgery. Am J Surg 139: 160-167, 1980.

6. Onodera T, Goseki N and Kosaki G: Prognostic nutritional index in gastrointestinal surgery of malnourished cancer patients. Nihon Geka Gakkai Zasshi 85: 1001-1005, 1984 (In Japanese).

7. Okada S, Shimada J, Kato D, Tsunezuka H, Teramukai S and Inoue M: Clinical significance of prognostic nutritional index after surgical treatment in lung cancer. Ann Thorac Surg 104 296-302, 2017.

8. Miao Y, Li S, Yan Q, Li B and Feng Y: Prognostic significance of preoperative prognostic nutritional index in epithelial ovarian cancer patients treated with platinum-based chemotherapy. Oncol Res Treat 39: 712-719, 2016.

9. Haraga J, Nakamura K, Omichi C, Nishida T, Haruma T, Kusumoto T, Seki N, Masuyama H, Katayama N, Kanazawa S and Hiramatsu Y: Pretreatment prognostic nutritional index is a significant predictor of prognosis in patients with cervical cancer treated with concurrent chemoradiotherapy. Mol Clin Oncol 5: 567-574, 2016.

10. Han WX, Chen ZM, Wei ZJ and Xu AM: Preoperative pre-albumin predicts prognosis of patients after gastrectomy for adenocarcinoma of esophagogastric junction. World J Surg Oncol 14: 279, 2016.

11. Yang Y, Gao P, Chen X, Song Y, Shi J, Zhao J, Sun J, Xu Y and Wang Z: Prognostic significance of preoperative prognostic nutritional index in colorectal cancer: Results from a retrospective cohort study and a meta-analysis. Oncotarget 7: 58543-58552, 2016.

12. Ke M, Xu T, Li N, Ren Y, Shi A, Lv Y and He H: Prognostic nutritional index predicts short-term outcomes after liver resection for hepatocellular carcinoma within the Milan criteria. Oncotarget 7: 81611-81620, 2016.

13. Chan AW, Chan SL, Wong GL, Wong VW, Chong CC, Lai PB, Chan HL and To KF: Prognostic nutritional index (pni) predicts tumor recurrence of very early/early stage hepatocellular carcinoma after surgical resection. Ann Surg Oncol 22: 4138-4148, 2015.

14. Yamada S, Shimada M, Morine Y, Imura S, Ikemoto T, Arakawa Y, Saito Y, Yoshikawa M and Miyazaki K: Significance of frailty in prognosis after hepatectomy for elderly patients with hepatocellular carcinoma. Ann Surg Oncol: Jun 19, 2020 (Epub ahead of print)
15. Yamada S, Morine Y, Imura S, Ikemoto T, Arakawa Y, Saito Y, Yoshikawa M, Miyazaki K and Shimada M: Prognostic prediction of apparent diffusion coefficient obtained by diffusion-weighted MRI in mass-forming intrahepatic cholangiocarcinoma. J Hepatobiliary Pancreat Sci 27: 388-395, 2020.

16. Balzan S, Belghiti J, Farges O, Ogata S, Sauvanet A, Delefosse D and Durand F: The '50-50 criteria' on postoperative day 5: An accurate predictor of liver failure and death after hepatectomy. Ann Surg 242: 824-829, 2005

17. Ji F, Liang Y, Fu S, Chen D, Cai X, Li S, Peng B, Liang L and Hua Y: Prognostic value of combined preoperative prognostic nutritional index and body mass index in HCC after hepatectomy. HPB (Oxford) 19: 695-705, 2017.

18. Peng W, Li C, Wen TF, Yan LN, Li B, Wang WT, Yang JY and $\mathrm{Xu}$ MQ: Postoperative prognostic nutritional index change is an independent predictor of survival in patients with small hepatocellular carcinoma. Am J Surg 212: 122-127, 2016.

19. Man Z, Pang Q, Zhou L, Wang Y, Hu X, Yang S, Jin H and Liu H: Prognostic significance of preoperative prognostic nutritional index in hepatocellular carcinoma: A meta-analysis. HPB (Oxford) 20: 888-895, 2018.

20. He CB and Lin XJ: Inflammation scores predict the survival of patients with hepatocellular carcinoma who were treated with transarterial chemoembolization and recombinant human type-5 adenovirus H101. PLoS One 12: e0174769, 2017.

21. Taussig MD, Irene Koran ME, Mouli SK, Ahmad A, Geevarghese S, Baker JC, Lipnik AJ, Banovac F and Brown DB: Neutrophil to lymphocyte ratio predicts disease progression following intra-arterial therapy of hepatocellular carcinoma. HPB (Oxford) 19: 458-464, 2017.

22. Shirabe K, Mano Y, Muto J, Matono R, Motomura T, Toshima T, Takeishi K, Uchiyama H, Yoshizumi T, Taketomi A, et al: Role of tumor-associated macrophages in the progression of hepatocellular carcinoma. Surg Today 42: 1-7, 2012.

23. Harimoto N, Shirabe K, Nakagawara H, Toshima $T$, Yamashita Y, Ikegami T, Yoshizumi T, Soejima Y, Ikeda T and Maehara Y: Prognostic factors affecting survival at recurrence of hepatocellular carcinoma after living-donor liver transplantation: With special reference to neutrophil/lymphocyte ratio. Transplantation 96: 1008-1012, 2013.

24. Peng W, Li C, Wen TF, Yan LN, Li B, Wang WT, Yang JY and $\mathrm{Xu}$ MQ: Neutrophil to lymphocyte ratio changes predict small hepatocellular carcinoma survival. J Surg Res 192: 402-408, 2014.

25. Koike Y, Miki C, Okugawa Y, Yokoe T, Toiyama Y, Tanaka K, Inoue Y and Kusunoki M: Preoperative C-reactive protein as a prognostic and therapeutic marker for colorectal cancer. J Surg Oncol 98: 540-544, 2008.

26. Okugawa Y, Toiyama Y, Yamamoto A, Shigemori T, Ide S, Kitajima T, Fujikawa H, Yasuda H, Hiro J, Yoshiyama S, et al: Lymphocyte-C-reactive protein ratio as promising new marker for predicting surgical and oncological outcomes in colorectal cancer. Ann Surg: Aug 2019 (Epub ahead of print).

27. Ohtani H, Mori K, Nakajima M and Hamaichi U: Defining lymphocyte-predominant breast cancer by the proportion of lymphocyte-rich stroma and its significance in routine histopathological diagnosis. Pathol Int 65: 644-651, 2015.

28. Lee YY, Choi CH, Sung CO, Do IG, Hub SJ, Kim HJ, Kim TJ, Lee JW, Bae DS and Kim BG: Clinical significance of changes in peripheral lymphocyte count after surgery in early cervical cancer. Gynecol Oncol 127: 107-113, 2012.

29. Yoon CI, Park S, Cha YJ, Lee HS, Bae SJ, Cha C, Lee DY, Ahn SG and Jeong QJ: Associations between absolute neutrophil count and lymphocyte-predominant breast cancer. Breast 50: $141-148,2020$.

30. Okadome K, Baba Y, Yagi T, Kiyozumi Y, Ishimoto T, Iwatsuki M, Miyamoto Y, Yoshida N, Watanabe M and Baba H: Prognostic nutritional index, tumor-infiltrating lymphocytes, and prognosis in patients with esophageal cancer. Ann Surg 271: 693-700, 2020.

31. Okabayashi T, Nishimori I, Sugimoto T, Maeda H, Dabanaka K, OnishiS,Kobayashi M and Hanazaki K: Effects of branched-chain amino acids-enriched nutrient support for patients undergoing liver resection for hepatocellular carcinoma. J Gastroenterol Hepatol 23: 1869-1873, 2008.

32. Okabayashi T, Nishimori I, Nishioka A, Yamashita K, Sugimoto T, Dabanaka K, Maeda H, Kohsaki T, Ogawa Y, Kobayashi M, et al: Long-term effects of multimodal treatment for patients with resectable carcinoma of the pancreas. Oncol Rep 20: 651-656, 2008. 